\title{
Adsorption Free Energy of Glycine on TiO2 in Water from First Principles Simulations and Electrochemical Impedance Spectroscopy
}

Lorenzo Agosta ( $\sim$ lorenzo.agosta@kemi.uu.se )

Uppsala University https://orcid.org/0000-0002-9778-5275

Luca Fiore

University of Rome Tor Vergata

Noemi Colozza

University of Rome Tor Vergata

Alexander Lyubartsev

Stockholm University https://orcid.org/0000-0002-9390-5719

\section{Fabiana Arduini}

Università di Roma Tor Vergata

Kersti Hermansson

Uppsala University

\section{Article}

Keywords: mino acids, Glycine, computational study, spectroscopy, TiO2 bridging oxygens.

Posted Date: December 22nd, 2021

DOI: https://doi.org/10.21203/rs.3.rs-1155823/v1

License: (9) (i) This work is licensed under a Creative Commons Attribution 4.0 International License.

Read Full License 


\title{
Adsorption free energy of Glycine on $\mathrm{TiO}_{2}$ in
} water from first principles simulations and electrochemical impedance spectroscopy.

\author{
Lorenzo Agosta, ${ }^{*, \dagger}$ Luca Fiore, ${ }^{\ddagger}$ Noemi Colozza,, \\ Alexander Lyubartsev, " Fabiana Arduini, ${ }^{\ddagger}$ and Kersti Hermansson ${ }^{\S}$ \\ †Department of Chemistry, Angström Laboratory, Uppsala University, 75121 Uppsala, \\ Sweden \\ $\ddagger$ Department of Science and Chemical Technologies, University of Rome Tor Vergata, Via \\ della Ricerca Scientifica, 00133 Rome, Italy \\ 【Department of Materials and Environmental Chemistry, Stockholm University, 10691 \\ Stockholm, Sweden \\ $\S$ Department of Chemistry-Ångström Laboratory, Uppsala University, 75121 Uppsala, \\ Sweden \\ E-mail: lorenzo.agosta@kemi.uu.se
}




\begin{abstract}
The adhesion of amino acids and small organic molecules on $\mathrm{TiO}_{2}$ nanoparticles is fundamental for bio-nano functionalization of peptides and proteins. The adsorption free energy is the main physical quantity that regulates the adsorption process. Its evaluation is particularly challenging both experimentally, due to the weak interfacial signal in aqueous environments, and by atomistic simulations, due to the complexity of the physical phenomena occurring at the solid-water interface (polarization and charge transfer effects). We report here an accurate experimental-computational study of hydrated $\mathrm{TiO}_{2}$ nanoparticles interacting with Glycine where we obtain quantitative agreement of the measured adsorption free energy. Ab-initio simulations are performed within the Tight Binding Density Functional Theory in combination with enhanced free energy sampling techniques. The experiments adopt a new and efficient set-up for electrochemical impedance spectroscopy measurements based on screen-printed gold electrodes. The measured adsorption free energy is about $-30 \mathrm{~kJ} / \mathrm{mol}$ (both from experiment and calculation), with preferential interaction of the charged $\mathrm{NH}_{3}$ group which strongly adsorbs on the $\mathrm{TiO}_{2}$ bridging oxygens. The perfect agreement between computation and experiment opens the doors to an extended exploration of the bionano interaction for different materials and molecules.
\end{abstract}

\title{
Introduction
}

Specific molecular recognition by inorganic materials is a central concept ${ }^{112}$ in nanodrug delivery ${ }^{3}$ and nanotoxic response, $\frac{4}{4}$ as well as for studies of penetrative and adhesive properties of adsorbing molecules. ${ }^{5}$ In all these cases the functional design of the application strongly depends on the chemical affinities between the adhering biomolecules and the inorganic material surfaces. $\frac{4|6| 7}{10}$ particular, nano-structured metal oxides play a fundamental role in this context due to their high concentration of surface reactive sites and their photo-catalytic power. ${ }^{8}$ 
In the present study we address the bio-adsorption on a metal-oxide surface in bulk water, namely the adsorption free energy of Glycine (Gly) on hydrated Titanium dioxide $\left(\mathrm{TiO}_{2}\right.$, anatase) nanoparticles (NPs). $\mathrm{TiO}_{2}$ is utilized in medicine ${ }^{9 \mid 10}$ for its high bio-compatibility, but the mechanisms that regulate the bio-adsorption on $\mathrm{TiO}_{2}$, and on metal oxides in general, are still unclear. It is known that NPs in biological fluids are coated with a corona of proteins that determines the cellular response. $\frac{11}{11}$ This corona links to the NPs only by a few small peptides. By phage display $\stackrel{112[13}{1}$ and $\mathrm{NMR}^{14}$ techniques it has been shown that charged amino acids like Arginine, Lysine and Aspartate drive the adsorption of small peptides on $\mathrm{TiO}_{2}$, with an estimated peptide adsorption free energy of $20-40 \mathrm{~kJ} / \mathrm{mol}$. When embedded in a peptide only the amino acid side chains play a role in the adsorption process, while for single amino acid molecules the interaction with a surface also includes the contribution of the amino and carboxyl terminal groups. For this reason extracting the contribution of amino acid side chains to the adhesion mechanism is particularly challenging. For instance, free energy measurements of amino acid molecular adsorption on $\mathrm{TiO}_{2}$ NPs using BET models (see, e.g., Ref. $\frac{[15}{}$ ) have given values in the range of $10-20 \mathrm{~kJ} / \mathrm{mol}$ for all 21 amino acids (even for the non-polar ones which is probably due to the interaction of the amino-carboxyl zwitterionic groups with the $\mathrm{TiO}_{2}$ surface).

Glycine is the only amino acid that merely possesses the amino and carboxyl groups (in their zwitterion state) making Glycine a perfect model amino acid molecule for investigating the bio-interaction with solids, as in the case of adsorption and polymerization of peptides on metal/metal-oxide surfaces. $\underline{16[17}$

Besides specific amino acid affinities with the solid, other factors can influence the adsorption of peptides onto metal-oxides surfaces. For instance, it has been observed that the lattice geometry of the exposed NPs facets can have a significant influence on the adsorption mode, $\frac{16|18| 19}{}$ while surface defects have been found to play a minor role. Moreover, the presence, or not, of water is crucial. An example is the anionic interactions of negatively charged amino acids which have been found to guide the peptide adhesion on dry surfaces, while it 
is not clear that this is the case in presence of water $\frac{18119}{1 n}$ fact, in aqueous environment, where most bio-applications operate, water molecules adsorb onto the under-coordinated metal and oxygen atoms at the surface and form a layer-like structure that mediates the interactions with the adsorbing molecules. $\frac{820}{20}$ The structure of this solid-liquid interface, including its protonation state, regulates the adhesion of molecules and it is thus fundamental for understanding the adsorption mechanism $\frac{51821}{}$ Finally, it should also be pointed out that, not least for long peptides, the configurational entropy and the specific amino acid sequence can play a fundamental role for the adsorption. ${ }^{22123}$

Our ability to resolve these details concerning the adsorption process strongly depends on the availability of reliable experimental measurements as well as modelling results. Atomistic simulations in principle represent a unique and powerful toolbox to unravel the complex physical phenomena that control the bio-molecular binding at metal-oxide water interfaces. Thus, for example, many classical (i.e. with force-fields) molecular dynamics simulations combined with free energy calculations for polar, charged and zwitterionic amino acids have been performed with the goal to establish their adsorption hierarchy on different wet $\mathrm{TiO}_{2}$ surfaces and provide insights concerning the adhesion mechanisms. ${ }^{244}$ These studies overall agree that charged amino acids demonstrate preferential adsorption over the polar/apolar amino acids, and that positively charged groups give rise to higher adsorption free energy values $(\approx 20 \mathrm{~kJ} / \mathrm{mol})$ than the negative ones $(\approx 10 \mathrm{~kJ} / \mathrm{mol})$. At the same time it should be noted that classical pair potentials tend to overestimate the water-titania interactions (see discussions in Ref. .33 and references therein). This is likely to imply overstructured interfacial water that would artificially hinder adsorption and diminish the adsorption free energy.

In the present work we study the adsorption behaviour of Glycine on the $\mathrm{TiO}_{2}$ anatase (101) surface by evaluating its adsorption free energy both computationally and experimentally. On the computational side we perform molecular dynamics simulations that retain the presence of the electrons, thereby allowing the description of polarization, reactivity and charge transfer effects. 
Free energy calculations based on electronic structure calculations generally require an unfeasible amount of computational time. However combining metadynamics techniques with thermodynamics integration can speed up the convergence of the free energy calculation by at least a factor $100^{\frac{34}{4}}$ compared to standard metadynamics. To the best of our knowledge the current paper is the first attempt to sample the Glycine free energy landscape by electronic methods.

The experimental part adopts a novel and quick approach to sample the Langmuir isotherm by electrochemical impedance spectroscopy (EIS) on screen-printed sensors. The results presented in this article show very nice agreement between the experimental and computational free energy evaluation and provide insights about the configurations of the binding modes (one in contact with the surface, one solvent-mediated). Our methodology should be extendable to other biomolecules and inorganic surfaces, opening up for a systematic investigation of the bio-inorganic interfaces.

\section{Method}

\section{Computational details}

All simulations were performed with the CP2 $\mathrm{K}^{[35}$ code coupled with the PLUMED software ${ }^{36}$ for Metadynamics. The results involve three types of electronic structure methodologies: MD simulations using Density Functional Tight-Binding ("DFTB-MD"), MD simulations using DFT ("DFT-MD"), Metadynamics simulations using DFTB ("DFTB-MetaDF"), and one single-point DFT and DFTB simulation for validation of the adsorption energy as summarised in Table 1 .

Self Consistent Charge Density Functional Tight-Binding approach ${ }^{37}$ was used with the Matsci parametrization ${ }^{38}$ for the atomic pair interactions. No long range corrections were used.

The simulation boxes were prepared with PACKMOL ${ }^{39}$ software. The box sizes were 
fixed at $10.55 \times 11.40 \times 43.00 \AA^{3}$. A slab of $\mathrm{TiO}_{2}$ anatase $(101)$ containing $4 \times 3 \times 3$ unit cells repetitions were used while the remaining space in the simulation box was filled with a Glycine molecule and water at its relative density for 1 atm and $310 \mathrm{~K}$ (see Fig. 11). $\frac{40}{\text { The }}$ systems were set to the temperature of $310 \mathrm{~K}$ by rescaling the velocities with the thermostat of Bussi $\frac{41}{4}$ and using an integration time step of 0.5 fs. A DFTB-MD simulation was run for 50 ps for a system containing only the $\mathrm{TiO}_{2}$ anatase (101) and water in order to evaluate the water structure at the interface for the Matsci parameters and compared with previously published DFT-MD data. $\underline{40}$

\section{Table 1: Simulations details}

\begin{tabular}{cccc} 
Method & Code & System & Simulation run and box size \\
\hline DFTB-MD & CP2K (Matsci) & $\mathrm{TiO}_{2}$-water & $50 \mathrm{ps}, 10.35 \times 11.08 \times 40.00 \AA^{3}$ \\
DFT-MD & CP2K (BLYP-D3) & $\mathrm{TiO}_{2}$-water & $50 \mathrm{ps}, 10.55 \times 11.40 \times 43.00 \AA^{3}$ \\
DFTB-MetaDF & CP2K (Matsci)-PLUMED & $\mathrm{TiO}_{2}$-water-Gly & $2.6 \mathrm{~ns}, 10.55 \times 11.40 \times 43.00 \AA^{3}$ \\
\hline
\end{tabular}

Metadynamics simulations were run within the well tempered diffusive approach (WTMetaD) 1424 with a bias factor of 15 . Gaussians with an initial height of $3.5 \mathrm{~kJ} / \mathrm{mol}$ were added every 25 fs and the widths were updated every 75 ps. These parameters were demonstrated to constitute a good compromise for ab-initio Metadynamics simulations, where the

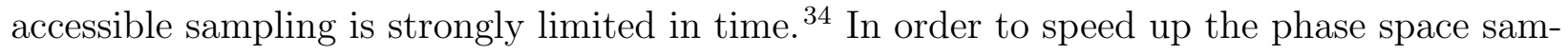
pling we made use of 8 walkers ${ }^{[45}$ starting from different initial configurations. A potential wall at $1 \mathrm{~nm}$ away from the outermost line of Ti atoms was introduced to restrict the sampled phase space. Each walker was run interchanging information with the remaining walkers every 25 fs. Glycine side chain consists of a single hydrogen atom and the amino-carboxyl groups are locally charged, $\mathrm{NH}_{3}{ }^{+}$and $\mathrm{COO}^{-}$, at physiological $\mathrm{pH}$. This is also the natural state predicted from ab-initio DFT simulations. The Matsci parameters make Glycine be stable in its neutral form. In order to maintain the amphoteric state we imposed a constraint on the $\mathrm{NH}$ and $\mathrm{CH}$ bond length to avoid deprotonation. 34

Two independent collective variables were considered for sampling the free energy land- 
scape. The surface separation distance (SSD) variable is defined as the distance between the outermost layer of $\mathrm{Ti}$ atoms on the $\mathrm{TiO}_{2}$ surface and the center of mass of the $\mathrm{NH}_{3}$ group in Glycine (Gly) amino acid (see Fig. 1)(b). Using the $\mathrm{NH}_{3}$ group instead of the center of the mass of the whole molecule allows to directly assess the adsorption modes. ${ }^{34}$ The second variable was defined as the angle between the normal vector to the $\mathrm{TiO}_{2}$ surface and the vector defined by the distance between $\mathrm{N}-\mathrm{C}_{\mathrm{N}}$ in Gly molecule $\left(\mathrm{C}_{\mathrm{N}}\right.$ is the first carbon neighbour atom to the Gly nitrogen 1)(b)). This variable was used in order to enhance the sampling of the phase space and it was integrated out in the calculation of the free energy. Up to 320 ps were simulated for each walker. The potential of the mean force (PMF) was calculated with the new method MetaDF ${ }^{34}$ that combines the Gaussians history ${ }^{29142}$ of the bias potential, and thermodynamic integration of the mean forces acting on the $\mathrm{NH}_{3}$ atoms along the collective variable SSD (see Fig. 1):

$$
\operatorname{PMF}_{\mathrm{TI}}(z)=-\int_{r_{c}}^{z}\left\langle F\left(z^{\prime}\right)\right\rangle \mathrm{d} z^{\prime}+\text { const }
$$

where $z$ spans the SSD-values from $r_{c}$ (the onset of the solid surface, to $1 \mathrm{~nm}$ (where the potential wall is set). $\langle F(z)\rangle$ is the canonical averaging of the average force weighted on the $2 \mathrm{D}$ bias potential. ${ }^{34}$ It has been shown that MetaDF converges in about 200-300 ps per walker for this set-up DFTB-MD simulation. ${ }^{34}$ The final binding free energy is computed as:

$$
\Delta G=-k T \ln \left(\frac{1}{\delta} \int_{r_{c}}^{r_{c}+\delta} e^{\frac{-P M F(z)}{k T}} d z\right)
$$

where $k_{B} T$ is the product of the Boltzmann constant and the absolute temperature, $\delta$ is the thickness of the adsorption layer and $r_{c}+\delta$ indicates the beginning of the liquid bulk.

We also performed a single-point DFT and DFTB calculation in order to compare the adsorption energy of the most stable Glycine binding mode on the dry $\mathrm{TiO}_{2}$ surface. The adsorption energy $E_{a d s}$ was calculated as $E_{a d s}=E_{\text {sup }+ \text { gly }}-E_{\text {sup }}-E_{\text {gly }}$ where $E_{\text {sup }}$ and $E_{\text {gly }}$ are the energies of two separated systems composed of the bare anatase (101) and Gly in the 
same position as in the adsorption mode that corresponds to $E_{\text {sup }+g l y}$. The DFT simulation was using the GGA-BLYP functional ${ }^{46147}$ with the Grimme D3 ${ }^{48}$ dispersion corrections. For further details we refer to Ref. $\underline{40}$

\section{Experimental details}

Commercial $\mathrm{TiO}_{2}$ nanopowder (Sigma-Aldrich 718467 ; $\geq 99.5 \%$ ) was used to coat the screenprinted electrodes. This powder is composed of particles of $21 \mathrm{~nm}$, a typical size for which the anatase phase is stable and exposes the (101) facet. ${ }^{15}$ As mentioned, for evaluating the adsorption of glycine on $\mathrm{TiO}_{2}$ anatase we adopted electrochemical impedance spectroscopy (EIS), a technique that can probe charge changes at the surface electrodes with high sensitivity and accuracy. By measuring the charge transfer resistance $\left(R_{c t}\right)$ of a redox process occurring between a solution of ferro-ferricyanide and the electrode surface it was possible to evaluate the amount of glycine adsorbed on the $\mathrm{TiO}_{2}$ powder. Herein, the Randles equivalent circuit ${ }^{49}$ was used as model for the electronic components of our sensor. A sinusoidal potential was applied to the electrochemical cell scanning a wide range of frequencies $(\Omega)$. Subsequently measurements of the resulting impedance $(Z(\Omega))$ were obtained. The $R_{c t}$ values were calculated from a Nyquist plot, $\frac{50]}{}$ which is composed of the imaginary part of $Z(\Omega)$ plotted as a function of the real part. The extrapolation of the semi-circular signal onto the x-axis of the Nyquist plot yields the $R_{c t}$ value.

Gold screen-printed electrodes (SPEs, S4M-PE08G) from Sense4Med company were used for the EIS experiments that were carried out with a portable potentiostat PalmSens3 (PalmSens Instrument), connected to a laptop equipped with PsTrace software.

For EIS measurements, the gold electrodes were modified by sequential drop-casting of 4 $\mu \mathrm{L}$ of a $\mathrm{TiO}_{2}$ dispersion $\left(\mathrm{TiO}_{2} 1 \mathrm{mg} / \mathrm{mL}\right)$ in distilled water, for a total amount of $12 \mu \mathrm{L}$. The modified sensors were allowed to dry on a hot plate at $100{ }^{\circ} \mathrm{C}$. Measurements were carried out in the range from $10000 \mathrm{~Hz}$ to $1 \mathrm{~Hz}$, with an increase of 10 points per frequency decade, thus scanning 41 frequencies. A potential of $0 \mathrm{~V}$ was applied to the electrochemical cell, 
with a potential amplitude of $0.01 \mathrm{~V}$. Increasing concentrations of glycine (i.e. from 1 to 300 $\mu \mathrm{M})$ were analyzed on the same sensor, by washing the electrode surface with distilled water after each measurement. The study was conducted using four independent electrodes.
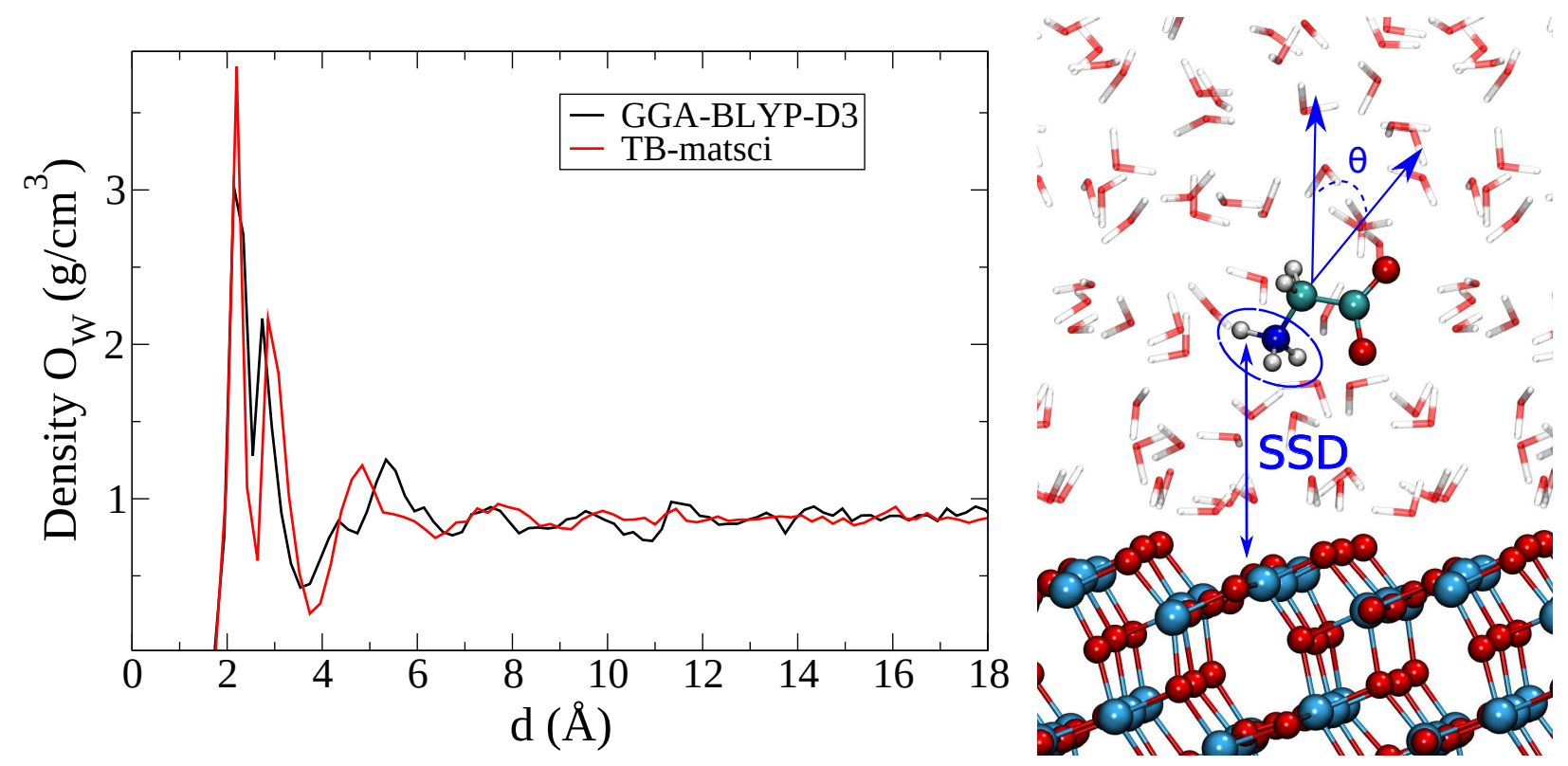

Figure 1: (left) Water oxygen density profiles for the MD simulations using TB-Matsci parameters and with DFT-GGA approach. The small differences between the first peaks in the profiles can be attributed to the difference in box size. (right) A representation of the hydrated Gly-anatase (101) system and the collective variables SSD and $\Theta$ for the Metadynamics simulation.

We note that the adsorption of Gly on the bare gold electrode yielded a $R_{c t}$ value that did not vary as function of the Gly concentration. Therefore we could discard the possibility that the electrode had affected the Gly adsorption process on $\mathrm{TiO}_{2}$ nanoparticles.

The adsorption free energy of Glycine on $\mathrm{TiO}_{2}$ was evaluated by assuming that $R_{c t}$ is proportional to the amount of Glycine adsorbed on the $\mathrm{TiO}_{2}$ nanoparticles deposited on the gold electrodes. Under this assumption it is possible to build a Langmuir isotherm where the $R_{c t}$ data are scaled to resemble the standard Langmuir equation $\underline{51}$

$$
A=\frac{R_{c t}-R_{c t_{\min }}}{R_{c t_{\max }}-R_{c t_{\min }}}=\frac{C}{C+\frac{1}{K}}
$$

where $A$ is a function of the charge transfer resistance $R_{c t}$, and it is proportional to the 
Gly surface coverage. $A$ varies in the range of $0-1 . C$ is the molar concentration of Glycine and $K$ is the equilibrium constant for the adsorption. Once $K$ is known, the adsorption free energy can easily be derived from

$$
\Delta G=-k T \ln (K)
$$

\section{Results and discussion}

Glycine is stable in its amphoteric form at neutral $\mathrm{pH}$ in aqueous solution. The adsorption on the $\mathrm{TiO}_{2}$ anatase (101) surface depends on the possibility for Gly to penetrate the layered water structure naturally forming at the very interface. ${ }^{26129134152}$ In Fig. 1 (black curve) the density of the water oxygen atoms along the surface normal is reported for the DFTB-MD simulation containing only the $\mathrm{TiO}_{2}$ slab and water molecules (without Gly) (cf. Table 1). The $\mathrm{x}$-axis $(d)$ denotes the distance between the water oxygen atoms and the outermost Ti atoms. For comparison, the water oxygen density profile of a hydrated anatase (101) surface simulated with the DFT-MD (BLYP) method, taken from Ref., $\frac{40}{45}$ also reported (red curve). It is evident that the two water structures at the interface are quite similar and that the DFTB Matsci parameters appear to reproduce the DFT-GGA features.

We can identify three water layers: water molecules adsorbed on the five-coordinated titanium surface atoms, $\mathrm{Ti}_{[5]}$, giving a density peak at $d \approx 2.3 \AA$, water hydrogen bonded to the bridging surface oxygens, $\mathrm{O}_{\mathrm{br}}$, at $d \approx 3.0 \AA$ and a third-layer of water molecules at $d \approx 5.0 \AA$. The small differences between the two methods, especially for the first peak in the density profile, can be accounted for by the different surface lattice geometries resulting from differences in the box sizes used in the two simulations (see Table 1). The Matsci parameters were constructed for water-titania interfaces ${ }^{\frac{38}{38}}$ but it is known that bulk water is not well described (the water-water interactions are underestimated). However, the free energy evaluation in MetaDF is based on the mean force acting on the adsorbing molecule. 
In the bulk region, the water density is uniform and isotropic, and thus Gly will feel an overall null mean force acting on it, which does not affect the evaluation of the free energy according to Eq. 1 .
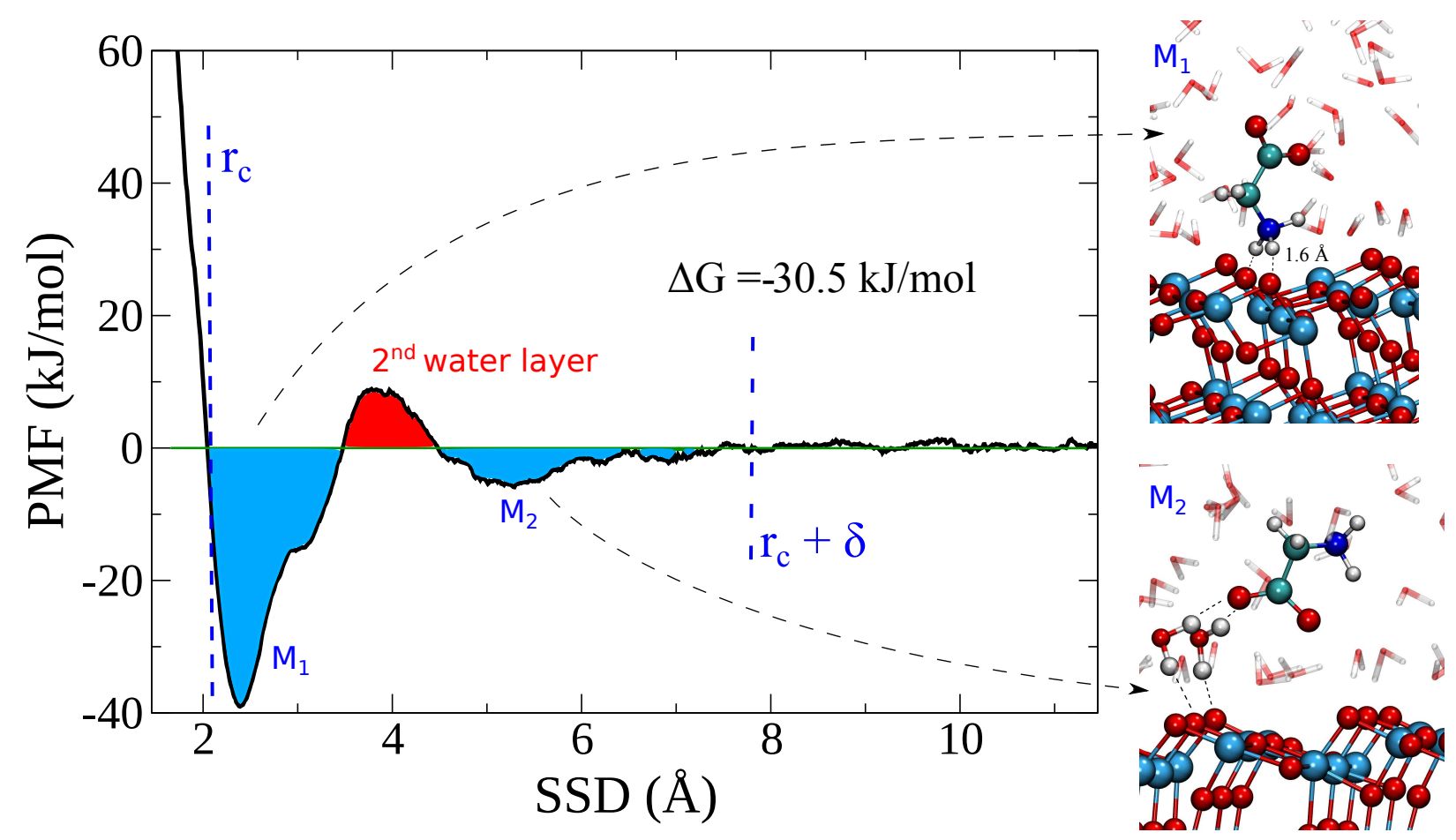

Figure 2: The Potential of the Mean Force (PMF) of Gly adsorbing on $\mathrm{TiO}_{2}$ anatase (101) surface. Two adsorption modes can be identified $M_{1}$ and $M_{2}$ separated by an energy barrier of $5 \mathrm{kj} / \mathrm{mol}$. $M_{1}$ represents the adsorption via the $\mathrm{NH}_{3}$ group to the $\mathrm{O}_{\mathrm{br}}$ atoms while $M_{2}$ occurs by adsorption to the second water layer. The total adsorption free energy is computed by eq. 2 using the illustrated $r_{c}$ and $\delta$ values.

The potential of the mean force calculated with MetaDF along the SSD variable is reported in Fig. 2 showing a converged adsorption profile after 300 ps (per walker) of simulation. Two main adsorption modes can be observed. The first one, $M_{1}$, consists of a direct bidentate interaction of the $\mathrm{NH}_{3}$ group with two $O_{b r}$ atoms which have lost their adsorbed waters. The amino group has been reported in ab-initio studies to be able to interact either via $O_{b r}$ or via the second layer of adsorbed water. $\frac{3453 \mid 54}{154}$ The free energy valley spans a range of $1.5 \AA$ from the surface, which approximately corresponds to the second water layer (cf. Fig. 1). The adsorption free energy calculated through Eq. 2 for this mode is $\approx-27 \mathrm{~kJ} / \mathrm{mol}$. The $\mathrm{NH}_{3}$ group must replace the water molecules adsorbed on the $O_{b r}$ sites, and this requires 
overcoming a free energy barrier of $\approx 5 \mathrm{~kJ} / \mathrm{mol}$ (red area in Fig. 1). This barrier lies within the second and third water layers (Fig. 1), a region which has been reported to possess restrained water dynamics, $\frac{52}{5}$ wich might impede the Glycine mobility at the interface.

The mean $\mathrm{H}-\mathrm{O}_{\mathrm{br}}$ distance of the $\mathrm{NH}-\mathrm{O}_{\mathrm{br}}$ bond was found to be $\approx 1.6 \AA$, which indicates the formation of a rather strong hydrogen bond. We calculated the adsorption energy of the $M_{1}$ mode in the absence of water using both the DFTB and DFT approaches, as mentioned in the methodology section. The resulting $E_{a d s}$ values are $120 \mathrm{~kJ} / \mathrm{mol}$ with both methods. The bidentate adsorption mode implies an adsorption energy of $60 \mathrm{~kJ} / \mathrm{mol}$ per $\mathrm{NH}-\mathrm{O}_{\mathrm{br}}$ bond, which is too large to be ascribed to a strong $\mathrm{NH}$... O hydrogen bond (or any H-bond for that matter). We speculate that surface polarization and charge transfer effects here contribute to enhancing the Gly- $\mathrm{TiO}_{2}$ interactions.

We now turn to the second adsorption mode, $M_{2}$. Classical MD studies have reported that the $\mathrm{COO}^{-}$group adsorbs onto the $\mathrm{Ti}_{[5]}$ site on the $\mathrm{TiO}_{2}$ rutile surface for Glycine ${ }^{\sqrt{26}}$ and acidic amino acids such as Aspartate and Glutamic acid ${ }^{30[32}$ while adsorption via the second water layer was found from ab-initio simulations for acidic amino acids on anatase. ${ }^{34 \mid 53}$ In the $M_{2}$ adsorption mode in the present study, the carboxylate group adsorbs "on the second water layer" with a relative small adsorption free energy of $\approx 8 \mathrm{~kJ} / \mathrm{mol}$. The total adsorption free energy is $-30.5 \mathrm{~kJ} / \mathrm{mol}$ (within an error of $1 \mathrm{~kJ} / \mathrm{mol}$ ) and it consists of the sum of the two adsorption modes $M_{1}$ and $M_{2}$ minus the free energy barrier between them.

In Fig. 3, the 2D accumulated bias potential is plotted for the SSD and $\Theta$ collective variables. It is well visible that the $M_{1}$ mode has a preferential orientation of about $60^{\circ}$ with respect to the surface normal. This is due to the bidentate adsorption of two hydrogen atoms on the $O_{b r}$ atoms and it indicates a strongly reduced mobility with respect to the bulk solution. The $M_{2}$ mode does not display a preferential adsorption orientation due to the fact that it occurs on the second water layer, where there are no orientational constraints.

In summary so far, our MetaDF simulation for Glycine on anatase (101) exhibits preferential adsorption via the positively charged $\mathrm{NH}_{3}$ group, and weak adsorption to the second 


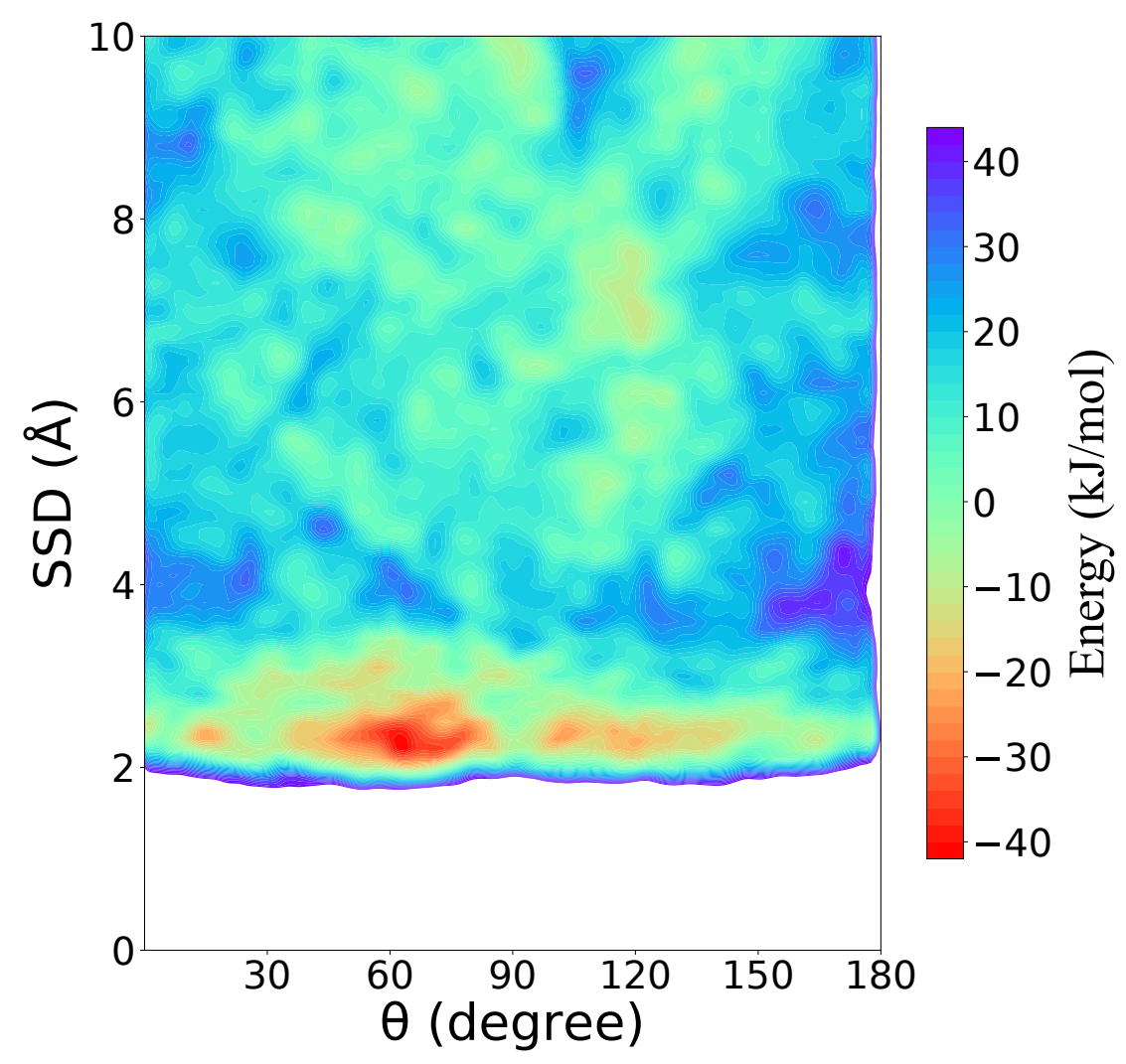

Figure 3: 2D plot of the accumulated bias potential for the SSD and $\Theta$ collective variables describing the Gly adsorbing on hydrated $\mathrm{TiO}_{2}$ anatase (101) surface.

water layer via the $\mathrm{COO}^{-}$group. The second water layer operates as an energy barrier for the $\mathrm{NH}_{3}$ adsorption and the overall adsorption free energy is $-30.5 \mathrm{~kJ} / \mathrm{mol}$, quite a lot larger than any previous value reported in literature.

On the experimental side, adsorption measurements for the hydrated Gly- $\mathrm{TiO}_{2}$ system were performed using the $\mathrm{TiO}_{2}$-modified gold screen-printed electrode (see Methodology section). In Fig. 4(b) the Langmuir isotherm is reported as the best fit of the four independent replicas used in the experimental set-up. The data match very well the Langmuir behaviour indicating that the Gly adsorption is indeed proportional to the $\mathrm{TiO}_{2}$ nanoparticles surface area and the $R_{c t}$. The derived adsorption free energy according to Eq. 3 and 4 is $-31 \pm 1$ $\mathrm{kJ} / \mathrm{mol}$. The experimental $\Delta G$ value perfectly matches the calculated one.

This is the first time that experimental and computational results for the adsorption free energy can be compared with such high accuracy, allowing not only to validate the techniques 

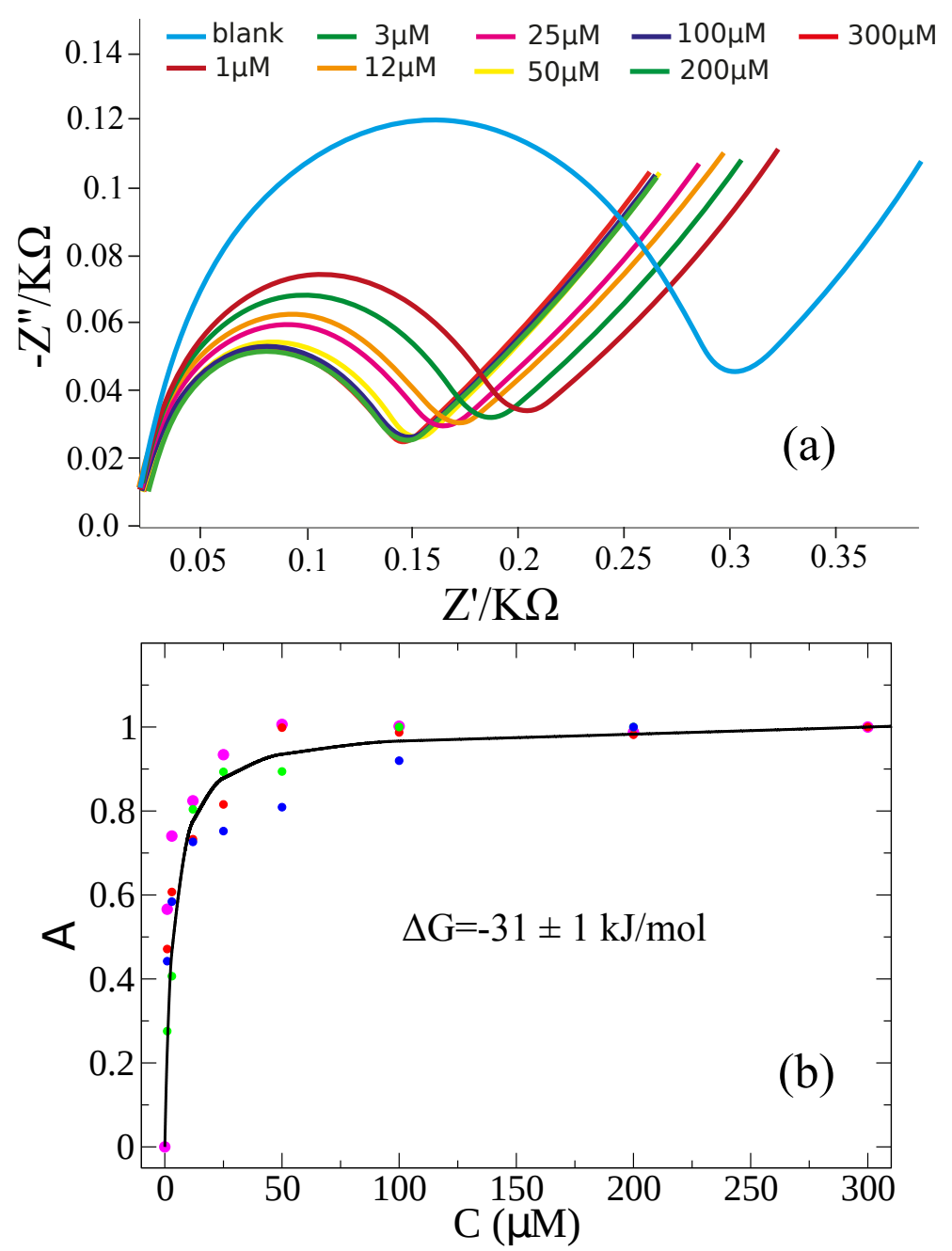

Figure 4: (a) Nyquist plot for the Gly adsorbing on the $\mathrm{TiO}_{2}$-modified gold screen-printed electrode. (b) Adsorption Langmuir isotherm for the hydrated $\mathrm{Gly}^{-\mathrm{TiO}_{2}}$ system. The fitted curve is obtain as the best fit of four different independent replicas. The calibration test of Glycine adsorbing on the bare gold electrode (without the $\mathrm{TiO}_{2}$ coating) produces constant $R_{c t}$ values in the concentration range of $1-300 \mu \mathrm{M}$, implying that the electrode does not affect the adsorption process.

implemented but also offering a new methodology for investigating the adsorption on solidliquid interfaces.

\section{Concluding remarks}

We have combined experimental and computational techniques in order to assess the adsorption thermodynamics of Glycine on the $\mathrm{TiO}_{2}$ anatase (101) surface. On the experimental 
side we introduced a new and quick method to construct a Langmuir isotherm from electrochemical impedance spectroscopy measurements on a screen-printed sensor from which accurate estimate of the adsorption free energy could be obtained. The principle of this sensor relies on the interaction of the glycine molecules adsorbed on the $\mathrm{TiO}_{2}$ nanoparticles deposited on the sensor surface, producing a glycine- $\mathrm{TiO}_{2}$ complex on the working electrode.

Electronic structure calculations were performed to properly describe the water structure at the interface and to take in consideration polarization and charged transfer effects.

The results show that Glycine adsorbs mainly through the charged amino group on the bridging oxygen of the anatase surface. The overall adsorption free energy is estimated to about $-30 \mathrm{~kJ} / \mathrm{mol}$ and it is the result of a balance between the energies of the adsorption modes and the energy barrier provided by the water structure at the interface. The large adsorption energy is mainly due to strong hydrogen bonding between the charged $\mathrm{NH}_{3}$ group and the $\mathrm{TiO}_{2}$ bridging oxygens. The methodologies presented in this paper can readily be extended to other biomolecules and nanoparticles for a systematic investigation of biointeractions on solid-liquid interfaces.

\section{Acknowledgements}

This work was supported by the INNOCONCRETE project "Innovative tools for conservation and monitoring of artworks in concrete by exploiting electrochemical screen-printed sensors, functionalized nanomaterials, and modelling", financed by the Italian Ministry of Education, University and Research (MIUR) within the Executive Program on Scientific and Technological Cooperation between the Italian Republic and Sweden, through the Ministry of Foreign Affairs and International Cooperation and the Swedish Research Council (VR). We also acknowledge financial support from the Swedish strategic collaborative research

programme in e-science, eSSENCE. The calculations described in this paper were performed using resources provided by the Swedish National Infrastructure for Computing (SNIC) at 
PDC.

\section{References}

(1) Shiba, K. Exploitation of peptide motif sequences and their use in nanobiotechnology. Curr. Op. Biotech. 2010, 21, 412-425.

(2) Willett, R. L.; Baldwin, K. W.; West, K. W.; Pfeiffer, L. N. Differential adhesion of amino acids to inorganic surfaces. Proceedings of the National Academy of Sciences 2005, 102, 7817-7822.

(3) Holmes, T. C.; de Lacalle, S.; Su, X.; Liu, G.; Rich, A.; Zhang, S. Extensive neurite outgrowth and active synapse formation on self-assembling peptide scaffolds. Proceedings of the National Academy of Sciences 2000, 97, 6728-6733.

(4) Khanna, P.; Ong, C.; Bay, B. H.; Baeg, G. H. Nanotoxicity: An Interplay of Oxidative Stress, Inflammation and Cell Death. Nanomaterials 2015, 5, 1163.

(5) Qian, J.; Gao, X.; Pan, B. Nanoconfinement-Mediated Water Treatment: From Fundamental to Application. Environ. Sci. Technol 2020, 54, 8509-8526.

(6) Wohlfart, S.; Gelperina, S.; Kreuter, J. Transport of Drugs Across the Blood-Brain Barrier By Nanoparticles. Journal of Controlled Release 2012, 161, 264-273.

(7) Lynch, I.; Salvati1, A.; Dawso, K. A. Protein-nanoparticle interactions: What does the cell see? Nature Nanotechnology 2009, 4, 546-547.

(8) Limo, M. J.; Sola-Rabada, A.; Boix, E.; Thota, V.; Westcott, Z. C.; Puddu, V.; Perry, C. C. Interactions between Metal Oxides and Biomolecules: from Fundamental Understanding to Applications. Chemical Reviews 2018, 118, 11118-11193. 
(9) Ziental, D.; Czarczynska-Goslinska, B.; Mlynarczyk, D.; Glowacka-Sobotta, A.; Stanisz, B.; Goslinski, T.; Sobotta, L. Titanium Dioxide Nanoparticles: Prospects and Applications in Medicine. Nanomaterials 2020, 10.

(10) Rajh, T.; Dimitrijevic, N. M.; Bissonnette, M.; Koritarov, T.; Konda, V. Titanium Dioxide in the Service of the Biomedical Revolution. Chemical Reviews 2014, 114, $10177-10216$.

(11) Lynch, I.; Feitshans, I. L.; Kendall, M. Bio-Nano Interactions: New Tools, Insights and Impacts: Summary of the Royal Society Discussion Meeting. Philos. Trans. R. Soc. Lond., B, Biol. Sci. 2015, 370, 20140162.

(12) Y. Fang, N. P.; Dickerson, M. B.; Cai, Y.; Jones, S. E.; Naik, R. R.; Kroger, N.; ; Sandhage, K. H. Identification of peptides capable of inducing the formation of titania but not silica via a subtractive bacteriophage display approach. J. Mater. Chem. 2008, 18, 3871-3875.

(13) Sano, K.-I.; Shiba, K. A Hexapeptide Motif That Electrostatically Binds To the Surface of Titanium. Journal of the American Chemical Society 2003, 125, 14234-14235.

(14) Suzuki, Y.; Shindo, H.; Asakura, T. Structure and Dynamic Properties of a Ti-Binding Peptide Bound to $\mathrm{TiO}_{2}$ Nanoparticles As Accessed by ${ }^{1} \mathrm{H}$ NMR Spectroscopy. The Journal of Physical Chemistry B 2016, 120, 4600-4607.

(15) Shchelokov, A.; Palko, N.; Potemkin, V.; Grishina, M.; Morozov, R.; Korina, E.; Uchaev, D.; Krivtsov, I.; Bol'shakov, O. Adsorption of Native Amino Acids on Nanocrystalline TiO2: Physical Chemistry, QSPR, and Theoretical Modeling. Langmuir 2019, 35, 538-550.

(16) Barcaro, G.; Sementa, L.; Carravetta, V.; Yano, T.-a.; Hara, M.; Monti, S. Experimental and theoretical elucidation of catalytic pathways in $\mathrm{TiO} 2$-initiated prebiotic polymerization. Phys. Chem. Chem. Phys. 2019, 21, 5435-5447. 
(17) Kitadai, N.; Oonishi, H.; Umemoto, K.; Usui, T.; Fukushi, K.; Nakashima, S. Glycine Polymerization on Oxide Minerals. Orig. Life Evol. Biosph. 2017, 47, 123-143.

(18) Costa, D.; Savio, L.; Pradier, C.-M. Adsorption of Amino Acids and Peptides on Metal and Oxide Surfaces in Water Environment: A Synthetic and Prospective Review. The Journal of Physical Chemistry B 2016, 120, 7039-7052.

(19) Costa, D.; Garrain, P.-A.; Baaden, M. Understanding small biomolecule-biomaterial interactions: A review of fundamental theoretical and experimental approaches for biomolecule interactions with inorganic surfaces. J Biomed Mater Res Part A 2013, $101 A, 1210-1222$.

(20) Barry, E. e. a. Advanced Materials for Energy-Water Systems: The Central Role of Water/Solid Interfaces in Adsorption, Reactivity, and Transport. Chemical Reviews 2021, 121, 9450-9501.

(21) YazdanYar, A.; Aschauer, U.; Bowen, P. Interaction of biologically relevant ions and organic molecules with titanium oxide (rutile) surfaces: A review on molecular dynamics studies. Colloids and Surfaces B: Biointerfaces 2018, 161, 563-577.

(22) Skelton, A. A.; Liang, T.; Walsh, T. R. Interplay of Sequence, Conformation, and Binding At the Peptide-Titania Interface As Mediated By Water. ACS Applied Materials $\&$ Interfaces 2009, 1, 1482-1491.

(23) Sultan, A. M.; Westcott, Z. C.; Hughes, Z. E.; Palafox-Hernandez, J. P.; Giesa, T.; Puddu, V.; Buehler, M. J.; Perry, C. C.; Walsh, T. R. Aqueous Peptide-TiO2 Interfaces: Isoenergetic Binding via Either Entropically or Enthalpically Driven Mechanisms. ACS Applied Materials \& Interfaces 2016, 8, 18620-18630.

(24) Monti, S.; Walsh, T. R. Free Energy Calculations of the Adsorption of Amino Acid Analogues at the Aqueous Titania Interface. The Journal of Physical Chemistry C 2010, 114, 22197-22206. 
(25) Schneider, J.; Ciacchi, L. C. Specific Material Recognition By Small Peptides Mediated By the Interfacial Solvent Structure. Journal of the American Chemical Society 2012, 134, 2407-2413.

(26) Li, C.; Monti, S.; Carravetta, V. Journey Toward the Surface: How Glycine Adsorbs on Titania in Water Solution. J. Phys. Chem. C 2012, 116, 18318-18326.

(27) Deighan, M.; Pfaendtner, J. Exhaustively Sampling Peptide Adsorption with Metadynamics. Langmuir 2013, 29, 7999-8009.

(28) Sultan, A. M.; Hughes, Z. E.; Walsh, T. R. Binding Affinities of Amino Acid Analogues at the Charged Aqueous Titania Interface: Implications for Titania-Binding Peptides. Langmuir 2014, 30, 13321-13329.

(29) Brandt, E. G.; Lyubartsev, A. P. Molecular Dynamics Simulations of Adsorption of Amino Acid Side Chain Analogues and a Titanium Binding Peptide on the $\mathrm{TiO}_{2}$ (100) Surface. The Journal of Physical Chemistry C 2015, 119, 18126-18139.

(30) YazdanYar, A.; Aschauer, U.; Bowen, P. Adsorption Free Energy of Single Amino Acids at the Rutile (110)/Water Interface Studied by Well-Tempered Metadynamics. The Journal of Physical Chemistry C 2018, 122, 11355-11363.

(31) Shengtang Liu, J. M. P.-A., Xuan-Yu Meng; Zhou, R. An In Silico study of TiO2 nanoparticles interaction with twenty standard amino acids in aqueous solution. Scientific Reports 2016, 6 .

(32) Janani Sampath, R. G. G. D., Andrew Kullman; Pfaendtner, J. Molecular recognition and specificity of biomolecules to titanium dioxide from molecular dynamics simulations. npj Comput. Mater. 2020, 6 .

(33) Liu, L.-M.; Zhang, C.; Thornton, G.; Michaelides, A. Reply to "Comment on 'Structure and dynamics of liquid water on rutile $\mathrm{TiO}_{2}(110)$ ' '. Phys. Rev. B 2012, 85, 167402. 
(34) Agosta, L.; Brandt, E.; Lyubartsev, A. Improved Sampling in Ab Initio Free Energy Calculations of Biomolecules at Solid-Liquid Interfaces: Tight-Binding Assessment of Charged Amino Acids on $\mathrm{TiO}_{2}$ Anatase (101). Computation 2020, 8 .

(35) Hutter, J.; Iannuzzi, M.; Schiffmann, F.; VandeVondele, J. CP2K: Atomistic Simulations of Condensed Matter Systems. WileyËnbsp;Interdisciplinary\&́nbsp;Reviews: Computational Molecular Science 2014, 4, 15-25.

(36) Tribello, G.; Bonomi, M.; Branduardi, D.; Camilloni, C.; Bussi, G. PLUMED2: New feathers for an old bird. Comp. Phys. Comm. 2014, 185.

(37) Elstner, M.; Porezag, D.; Jungnickel, G.; Elsner, J.; Haugk, M.; Frauenheim, T.; Suhai, S.; Seifert, G. Self-consistent-charge density-functional tight-binding method for simulations of complex materials properties. Phys. Rev. B 1998, 58, 7260-7268.

(38) Luschtinetz, R.; Frenzel, J.; Milek, T.; Seifert, G. Adsorption of Phosphonic Acid At the $\mathrm{TIO}_{2}$ Anatase (101) and Rutile (110) Surfaces. J. Phys. Chem. C 2009, 113, 57305740 .

(39) Martínez, L.; Andrade, R.; Birgin, E. G.; Martínez, J. M. Packmol: A package for building initial configurations for molecular dynamics simulations. Journal of Computational Chemistry 2009, 30(13), 2157-2164.

(40) Agosta, L.; Brandt, E. G.; Lyubartsev, A. P. Diffusion and reaction pathways of water near fully hydrated TiO2 surfaces from ab initio molecular dynamics. The Journal of Chemical Physics 2017, 147, 024704.

(41) Bussi, G.; Donadio, D.; Parrinello, M. Canonical Sampling Through Velocity Rescaling. The Journal of Chemical Physics 2007, 126, 014101.

(42) Barducci, A.; Bussi, G.; Parrinello, M. Well-Tempered Metadynamics: A Smoothly Converging and Tunable Free-Energy Method. Phys. Rev. Lett. 2008, 100, 020603. 
(43) Valsson, O.; Tiwary, P.; Parrinello, M. Enhancing Important Fluctuations: Rare Events and Metadynamics from a Conceptual Viewpoint. Annual Review of Physical Chemistry 2016, 67, 159-184.

(44) Branduardi, D.; Bussi, G.; Parrinello, M. Metadynamics with Adaptive Gaussians. Journal of Chemical Theory and Computation 2012, 8, 2247-2254.

(45) Raiteri, P.; Laio, A.; Gervasio, F. L.; Micheletti, C.; Parrinello, M. Efficient Reconstruction of Complex Free Energy Landscapes by Multiple Walkers Metadynamics. The Journal of Physical Chemistry B 2006, 110, 3533-3539.

(46) Becke, A. D. Density-Functional Exchange-Energy Approximation With Correct Asymptotic Behavior. Phys. Rev. A 1988, 38, 3098-3100.

(47) Lee, C.; Yang, W.; Parr, R. G. Development of the Colle-Salvetti Correlation-Energy Formula Into a Functional of the Electron Density. Phys. Rev. B 1988, 37, 785-789.

(48) Grimme, S. Semiempirical GGA-Type Density Functional Constructed With a LongRange Dispersion Correction. Journal of Computational Chemistry 2006, 27, 17871799.

(49) Chang, B.-Y.; Park, S.-M. Electrochemical Impedance Spectroscopy. Annual Review of Analytical Chemistry 2010, 3, 207-229.

(50) Peer Reviewed: Electrochemical Impedance Spectroscopy for Better Electrochemical Measurements. Analytical Chemistry 2003, 75, 455 A-461 A.

(51) Tamerler, C.; Oren, E. E.; Duman, M.; Venkatasubramanian, E.; Sarikaya, M. Adsorption Kinetics of an Engineered Gold Binding Peptide by Surface Plasmon Resonance Spectroscopy and a Quartz Crystal Microbalance. Langmuir 2006, 22, 7712-7718. 
(52) Agosta, L.; Dzugutov, M.; Hermansson, K. Supercooled liquid-like dynamics in water near a fully hydrated titania surface: Decoupling of rotational and translational diffusion. The Journal of Chemical Physics 2021, 154, 094708.

(53) Agosta, L.; Zollo, G.; Arcangeli, C.; Buonocore, F.; Gala, F.; Celino, M. Water Driven Adsorption of Amino Acids on the (101) Anatase $\mathrm{TiO}_{2}$ Surface: An Ab Initio Study. Phys. Chem. Chem. Phys. 2015, 17, 1556-1561.

(54) Pantaleone, S.; Rimola, A.; Sodupe, M. Canonical, Deprotonated, or Zwitterionic? A Computational Study on Amino Acid Interaction with the TiO2 (101) Anatase Surface. The Journal of Physical Chemistry C 2017, 121, 14156-14165. 